\title{
Familienersatz Pflegepersonal
}

\author{
Pflegeeinrichtungen und die Corona-Krise "Ich kann meine Mutter zurzeit \\ nur am Fenster ihrer Pflegeeinrichtung besuchen. Sie leidet an Demenz und \\ versteht deshalb gar nicht, warum ich nicht reinkommen kann", erzählt Ulrike \\ M. aus Ostwestfalen-Lippe. So erlebten viele in den vergangenen Wochen den \\ Kontakt zu ihren hochaltrigen oder pflegebedürftigen Angehörigen. Eine \\ Situationsbeschreibung, Stand Anfang Mai.
}

V on Mitte März bis Ende April galten bundesweit weitgehende Besuchsverbote in Pflegeeinrichtungen, um die Ansteckungswelle mit Covid-19 einzudämmen. Seit Mai werden die Regelungen nach und nach gelockert. Die Bewohner von Alten- und Pflegeeinrichtungen gehören aufgrund ihres Alters und/ oder ihrer Vorerkrankungen zu dem Personenkreis mit erhöhtem Risiko für einen schweren Krankheitsverlauf. Zudem ist wegen der gemeinsamen Unterbringung, der Teilnahme an gemeinschaftlichen Aktivitäten und dem körperlichen Kontakt zu den Pflegenden die Ansteckungsgefahr sehr hoch. 87\% der Todesfälle im Zusammenhang mit Covid-19 betreffen Menschen über 70 Jahre, die wiederum 19\% aller Fälle ausmachen, so die Zahlen Anfang Mai. Die Bewohner von Pflegeeinrichtungen müssen folglich besonders geschützt werden - aber auch die Pflegenden und Betreuenden. Die Bundesregierung, etliche Landesregierungen sowie viele deutsche Gesundheitsämter verfügten bis Redaktionsschluss Anfang Mai nicht über systematisch erhobene Zahlen der Corona-Infektionen bei medizinischem Personal.

Viele Bewohner von Pflegeeinrichtungen sind also von der Außenwelt abgeschnitten, ihre Welt ist begrenzt auf die Einrichtung. Kontakte zu Hausärzten, Friseuren, Freunden und Angehörigen sind auf ein Minimum reduziert oder gar nicht möglich. Gemeinschaftsveranstaltungen in den Einrichtungen sind eingeschränkt, Ausflüge außer Haus finden nicht statt. Gleichzeitig gelten in den Häusern strengere Hygieneregeln und damit auch ein neuer Umgang mit dem Pflegepersonal.

\section{Mit sachlicher Information aufklären}

In der Pflegeeinrichtung Kursana Villa Frankfurt am Main ist das Virus bisher nicht aufgetreten. 100 Menschen leben in der Einrichtung, sie sind im Durchschnitt etwa 82 Jahre alt und werden von 77 Mitarbeitern gepflegt und betreut. „Wir haben sehr schnell reagiert“, berichtet Pflegedienstleiterin Irina Sparwasser. „Bereits im März, noch vor dem offiziellen Besuchsverbot, haben wir die Einrichtung für Besuche geschlossen und unsere Bewohner angehalten, das Haus nicht zu verlassen. Bei einigen wenigen stieß das zunächst auf Unverständnis. Dann haben wir mit Informationsver- anstaltungen aufgeklärt, um einerseits Panik zu vermeiden und andererseits auch die Skeptiker ins Boot zu holen. Mittlerweile sind alle froh über unser frühes, konsequentes Vorgehen." Jeden Tag findet in der Kursana Villa ein Presseklub statt, der über den Verlauf der Pandemie aufklärt.

„Wir haben außerdem gleich zu Beginn das Personal geschult, zum Beispiel zum Thema Hygiene. Das hat dazu beigetragen, dass die Mitarbeiter gut vorbereitet waren und weniger Ängste hatten“, so Irina Sparwasser weiter. Seitdem werden die Flächen doppelt so oft pro Tag desinfiziert, es gibt Handschuhe, Schutzkleidung und ausreichend Desinfektionsmittel. „Natürlich gab es Engpässe bei der Beschaffung, aber letztendlich haben wir ausreichend Ausrüstung und Material bekommen." Als Vorteil hat sich die Struktur der Belegung in der Einrichtung erwiesen. Denn die Bewohner leben in verschiedenen Bereichen mit Stammpersonal, das nicht hinund herwechselt.

\section{Vom Fensterbesuch bis zum Haarefärben}

Die Bewohner haben aktuell mehrere Möglichkeiten, Kontakt mit der Außenwelt zu halten. In der Einrichtung gibt es zwei Tablets, die die Bewohner für Skype- oder Zoom-Sitzungen nutzen können.

$+$

\section{BETROFFENE VON COVID-19 IN DEUTSCHLAND}

Insgesamt wurden in Deutschland 162.469 laborbestätigte Covid-19-Fälle an das RKI übermittelt, darunter 6.649 Todesfälle in Zusammenhang mit Covid-19-Erkrankungen. $67 \%$ der an Covid-19 Erkrankten sind zwischen 15 und 50 Jahren alt. 87\% der Todesfälle und $19 \%$ aller Fälle betreffen Menschen über 70 Jahre. Von den Verstorbenen sind 58\% Männer. Der Altersmedian der Verstorbenen liegt bei 82 Jahren.

(Stand: 3.5.2020, Quelle RKI) 
+ LEITFADEN FÜR

\section{PFLEGEEINRICHTUNGEN}

Das Robert Koch-Institut (RKI) hat einen Leitfaden zu Prävention und Management von Covid-19 in Altenund Pflegeeinrichtungen und Einrichtungen für Menschen mit Beeinträchtigungen herausgegeben. Der Leitfaden ist abrufbar unter: rki.de (Suchwort Pflegeeinrichtungen)

Das Pflegepersonal begleitet sie dabei. Irina Sparwasser erzählt: „Unsere Bewohner sind technisch fit. Viele haben einen PC oder Laptop, die meisten haben ein Smartphone.“ Ferner gibt es „Fensterbesuche“. Diejenigen, die kognitiv oder motorisch nicht mehr in der Lage sind, diese Besuche allein zu bewältigen, bekommen Hilfe. Termine gibt es vormittags und nachmittags.
Technischer Support, Begleitung zu Besuchen am Fenster - das sind alles Aufgaben, die die Mitarbeiter jetzt leisten. Wie ist das zeitlich möglich? „Gemeinschaftsveranstaltungen finden kaum noch statt. Stattdessen gibt es Zeit für Einzelbeschäftigung. Dazu gehören die Fensterbesuche, aber auch tröstende Worte. Unsere Mitarbeiter frisieren, färben Haare und föhnen - der Friseur kommt ja nicht mehr. Oder sie gehen einkaufen, wenn einer der Bewohner mal was Spezielles benötigt. Wir versuchen, Wünsche zu erfüllen." Irina Sparwasser fährt fort: „Es gibt eigentlich für alle diese Dinge mehr Zeit. Das Leben ist jetzt weniger getaktet, niemand muss zu bestimmten Terminen fertig sein, dadurch ist alles entspannter.“

Die gelernte Altenpflegerin weiß ihr Team zu schätzen: „Durch diese Situation hat sich gezeigt, dass wir wie eine Familie sind. Der Zusammenhalt ist riesig, alle nehmen sich Zeit. Die Mitarbeiter versuchen, den Bewohnern ihre Angehörigen zu ersetzen. Sie sind unheimlich engagiert, viele bleiben von sich aus länger. Sowohl die Geschäftsführung als auch viele Angehörige haben sich dafür bereits bedankt.“ Sie überlegt kurz und ergänzt: „Wissen Sie, endlich erfahren wir in unserem Beruf die Anerkennung, die uns immer

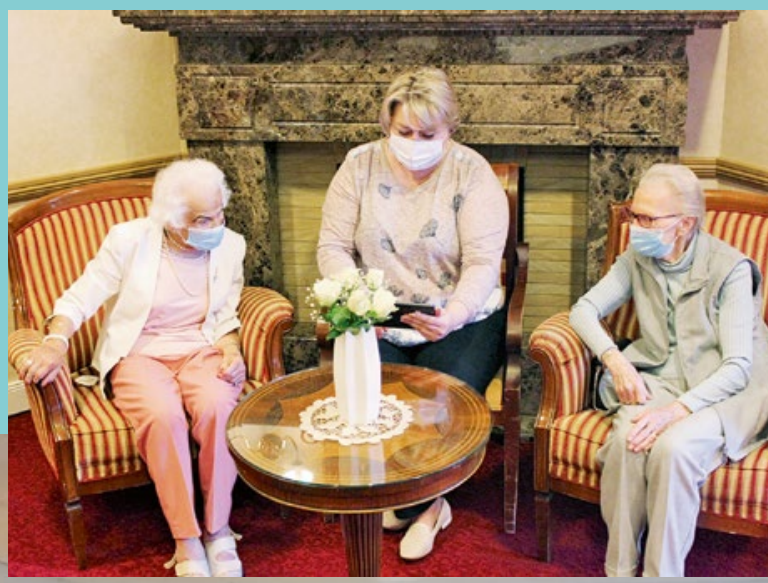

Kursana Villa Frankfurt am Main: Pflegedienstleiterin Irina Sparwasser mit zwei Bewohnerinnen
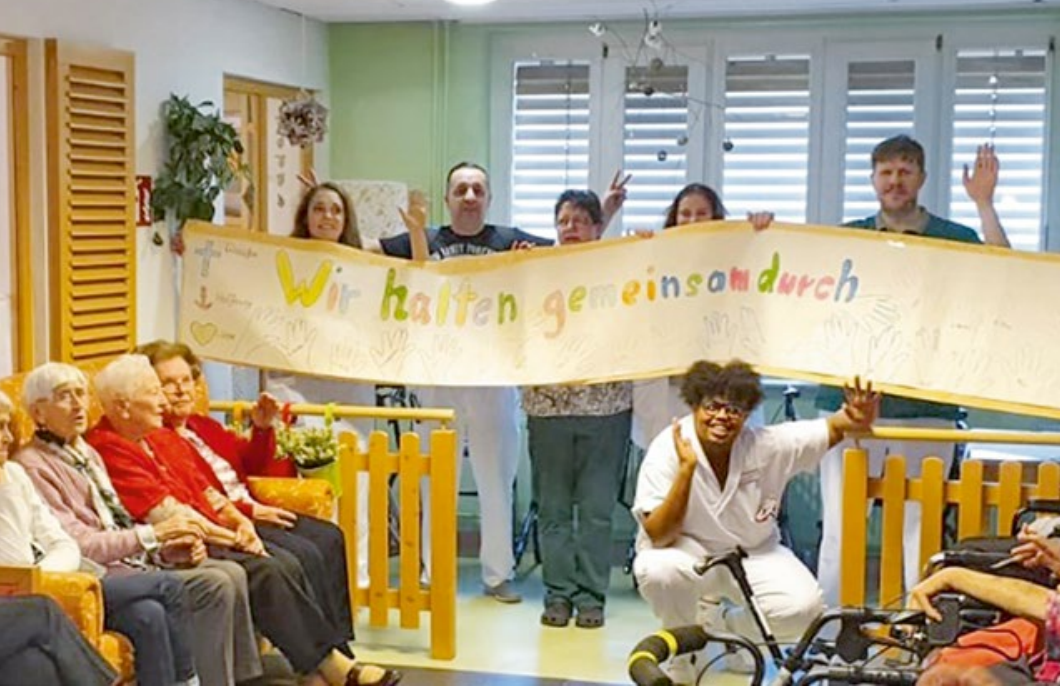
einsaludureh zweil zwe
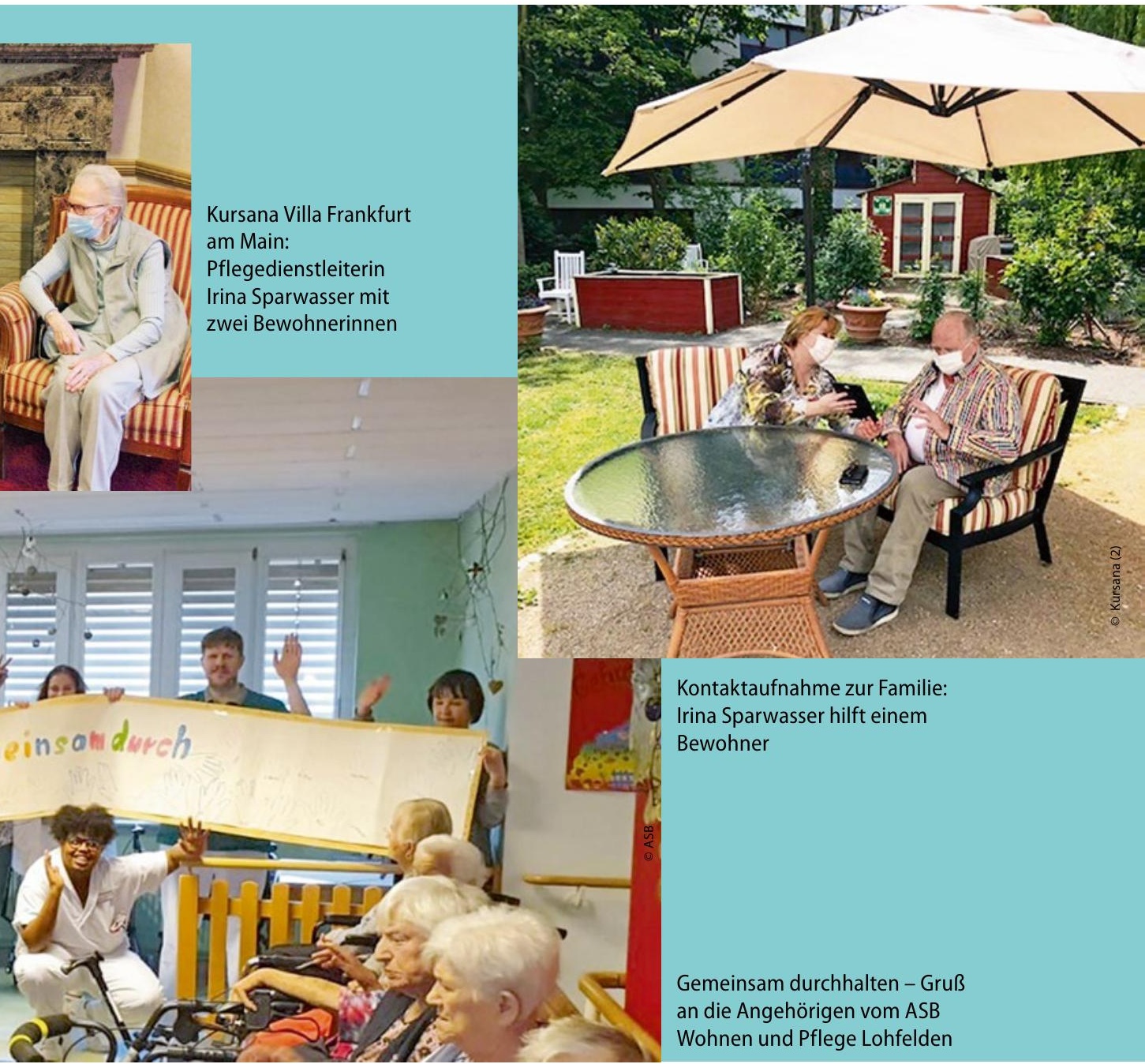
fehlte. Ich hoffe, dass das alles zu einem höheren Ansehen der Pflegeberufe in der Öffentlichkeit führt", so Irina Sparwasser.

\section{Endlich Anerkennung!}

Auf dieser Welle der Anerkennung reiten auch die Mitarbeiter der Einrichtung ASB Wohnen und Pflege Lohfelden. „Unser Team erfährt intern und extern im Moment sehr viel Wertschätzung“, sagt Einrichtungsleiterin Ute Schwendt. Und die benötigt das Team auch dringend, denn hier kämpfen die Mitarbeiter nicht nur mit den neuen Vorschriften und Vorsichtsmaßnahmen, sondern auch gegen die Ausbreitung des Virus. In der Einrichtung waren Stand Ende April acht der insgesamt 131 Bewohner mit einem Durchschnittsalter von etwa 85 Jahren infiziert und sieben Mitarbeiter von 142 .

\section{Infektion in der Einrichtung eindämmen}

Ein Mangel an Schutzkleidung und Ausrüstung besteht auch hier nicht. Seit Mitte März kauft der Träger ASB zentral ein, und da noch nicht viele Häuser betroffen sind, sind genug Handschuhe, Schutzmasken sowie Hauben und Schuhe für den Vollschutz da. Apotheken haben Desinfektionsmittel hergestellt, das lokale Sanitätshaus hat Ausrüstung für den Vollschutz schnellstens geliefert.

Die Abläufe in der Einrichtung sind jedoch völlig neu. „Wir alle haben technisch einen Riesensprung gemacht“, berichtet Ute Schwendt. „Wir haben täglich Telefon- und Videokonferenzen.“ Bis zehn Uhr muss sie jeden Tag dem Gesundheitsamt den aktuellen Stand durchgeben - das sind die Zahlen der Erkrankten, die auch in die Statistik der Gesundheitsämter eingehen, die jeder TV-Zuschauer abends in den Nachrichten sieht. „Nach einer Richtlinie des Robert Koch-Instituts fallen wir in die Kategorie ,Beobachten', das heißt, es wird eigentlich nur bei Symptomen getestet“, erklärt die Einrichtungsleiterin. „Mithilfe von Hausärzten im Umkreis von Lohfelden und Angehörigen konnten wir aber so viel Druck ausüben, dass jetzt alle in unseren beiden Häusern getestet werden. Das ist eine große Erleichterung, besonders für die Mitarbeiter. Die haben ja zu Hause auch Familien, die sie schützen wollen. “Die infizierten Bewohner stehen zusätzlich gesondert unter Quarantäne, also von den anderen getrennt. Sie ziehen jetzt um auf eine Station, zu der nur Personal in Vollschutz Zugang hat. Brandschutztüren riegeln diesen Trakt dann noch zusätzlich ab, ermöglichen dadurch eine begrenzte Bewegungsfreiheit der infizierten Bewohner, die zuvor ihr Zimmer nicht verlassen durften. „Das Team ist unglaublich“, sagt Ute Schwendt und kämpft mit den Tränen. „Viele haben ihren bereits genommenen Urlaub verschoben oder verzichten auf Urlaubstage, machen Nachtschichten und Sonderdienste.“

\section{Mal schnell den Sohn anrufen}

Da die gesamte Einrichtung unter Quarantäne steht, sind Besuche von Angehörigen auch an Fenstern nicht möglich. „Wir verschicken ein wöchentliches Update an die Angehörigen. Außerdem gibt es Videotelefonie oder Skype. Dabei helfen die Betreuungskräfte und der Sozialdienst. Wir helfen auch dabei, Fotos zu verschicken oder rufen mal eben schnell den Sohn an“, erzählt Ute Schwendt. „Wir haben hier ältere Damen, die zuvor fast rund um die Uhr von ihren Ehemännern begleitet wurden. Die dürfen jetzt nicht mehr kommen. Das ist für alle Beteiligten sehr belastend." Durch die gute

\section{+ POSTKARTEN-AKTION}

\author{
Die Bewohner in Pflegeeinrichtungen sind durch \\ Corona von der Außenwelt abgeschnitten, ein Projekt \\ der Caritas hält dagegen. Verschiedene Ortsverbände \\ rufen dazu auf, Briefe an Menschen in Pflegeeinrich- \\ tungen zu schreiben und ihnen damit den Alltag er- \\ träglicher zu machen. Dazu gehört zum Beispiel das \\ Caritas Zentrum in Attendorn, das sich über Facebook \\ an die Community wandte, und auch die youngcaritas \\ in Berlin. Hier kann man sich melden und bekommt \\ dann Kontaktdaten vermittelt: youngcaritas.de/berlin
}

Ausstattung mit Schutzausrüstung und die räumliche Aufteilung ist es jedoch möglich, dass Angehörige kommen können, um sich von Sterbenden zu verabschieden. Für sie steht Schutzkleidung bereit.

Die Mitarbeiter geben ihr Bestes, den Bewohnern den Alltag dennoch abwechslungsreich zu gestalten. Pflegedienstleiter Shaban Asani ergänzt: „Sie lesen aus der Zeitung vor oder der Pfleger zückt sein Handy und fotografiert die Bewohnerin, wie sie am Fenster steht und winkt, um das Foto dann Angehörigen zu schicken. "Wie in Frankfurt, fällt auch in Lohfelden der Vergleich mit der Familie. $\mathrm{Zu}$ Ostern gab es Sonderrezeptionszeiten, zu denen die Angehörigen Geschenke bei „Miss Easy“, der stets freundlichen Dame an der Rezeption, die für alle ein offenes Ohr hat, abgeben konnten. „Viele haben auch Geschenke für das Personal gebracht", sagt Shaban Asani. Eine Geste, die alle sehr zu schätzen wussten.

\section{Netzwerken zahlt sich aus}

„Ich empfinde es nicht so, dass wir jetzt komplett nach außen abgeschottet sind. Im Gegenteil, wir sind und waren immer gut vernetzt und das ist jetzt ein Riesenvorteil. Wir bekommen Schutzmasken gespendet, eine Ehrenamtliche, die zurzeit nicht kommen darf, hat ein Tablet gespendet. Der Heimbeirat hat Desinfektionsmittel organisiert. Die Ehrenamtlichen halten Kontakt über Skype oder rufen an. Hausarztpraxen haben uns angeboten, mit den Tests zu helfen. Und wir sind unsererseits offen für Fragen anderer Häuser, die mit der Situation überfordert sind“, so Ute Schwendt. „Wir wurden auch von den Ämtern gut begleitet, die kennen im Moment auch keine Wochenenden. Vom Gesundheitsamt kam zum Beispiel jemand zur Beratung ins Haus.“

III

Dr. Claudia Sabic 